\title{
Ataxias and Cerebellar or Spinocerebellar Degeneration
}

National Institute of Neurological Disorders and Stroke (NINDS)

\author{
Source \\ National Institute of Neurological Disorders and Stroke (NINDS). Ataxias and Cerebellar \\ or Spinocerebellar Degeneration Information Page.
}

Ataxia often occurs when parts of the nervous system that control movement are damaged. People with ataxia experience a failure of muscle control in their arms and legs, resulting in a lack of balance and coordination or a disturbance of gait. While the term ataxia is primarily used to describe this set of symptoms, it is sometimes also used to refer to a family of disorders. It is not, however, a specific diagnosis.

Most disorders that result in ataxia cause cells in the part of the brain called the cerebellum to degenerate, or atrophy. Sometimes the spine is also affected. The phrases cerebellar degeneration and spinocerebellar degeneration are used to describe changes that have taken place in a person's nervous system; neither term constitutes a specific diagnosis. Cerebellar and spinocerebellar degeneration have many different causes. The age of onset of the resulting ataxia varies depending on the underlying cause of the degeneration.

Many ataxias are hereditary and are classified by chromosomal location and pattern of inheritance: autosomal dominant, in which the affected person inherits a normal gene from one parent and a faulty gene from the other parent; and autosomal recessive, in which both parents pass on a copy of the faulty gene. Among the more common inherited ataxias are Friedreich's ataxia and Machado-Joseph disease. Sporadic ataxias can also occur in families with no prior history.

Ataxia can also be acquired. Conditions that can cause acquired ataxia include stroke, multiple sclerosis, tumors, alcoholism, peripheral neuropathy, metabolic disorders, and vitamin deficiencies. 